\title{
Transmission and Sensing Characteristics of the Biconically Tapered Cladded Multimode Fibers
}

\author{
Kwang Taek Kim* and Ki Bum Hong \\ Division of Electronic and Photonic Engineering, Honam University 59-1, \\ Seobong-Dong, Gwangsan-Gu, Gwangju 505-714, Korea \\ Jaehee Park \\ Department of Electronic Engineering, Keimyung University 1000 Sindang-Dong, \\ Dalseo-Gu, Daegu 704-701, Korea
}

(Received December 19, 2008 : revised April 20, 2009 : accepted April 20, 2009)

\begin{abstract}
This paper presents a theoretical and experimental investigation of the transmission and sensing characteristics of the biconically tapered cladded multimode fibers. The beam propagation method was used to examine the transmission characteristics with various structural parameters. The results show that the transmission of the biconically tapered cladded multimode fibers is sensitive to the mode of the input optical beam and the refractive index of the external medium. A refractive index sensor for the external medium was proposed based on the theoretical analysis, and its feasibility was demonstrated experimentally.
\end{abstract}

Keywords: Multimode fiber taper, Fiber-optic refractive index sensor, Beam propagation method

OCIS codes : (060.2310) Fiber optics; (060.2340) Fiber optic components; (060.2370) Fiber optic sensor; (060.2280) Fiber design and fabrication

\section{INTRODUCTION}

Optical fibers are applied widely in sensor systems because they can be used simultaneously as both an optical signal path and sensor. In particular, claddingremoved multimode fibers may interact directly with the external medium through an evanescent wave in the core-exposed region of a multimode fiber. Its device applications in biosensors, gas sensors and chemical sensors have been studied extensively.[1-4] Several authors have proposed the tapering of multimode fibers as a means of improving the sensitivity.[5-6]. Tapering of the multimode fiber causes an increase in the evanescent wave, resulting in an increase in sensitivity to the external medium. However, removal of the cladding of silica multimode fibers is not easy due to the accompanying etching process that requires careful control and the use of harsh chemicals. In addition, the tapering of etched fibers is very difficult because the etched region is more fragile.

*Corresponding author: ktkim@honam.ac.kr
The above-mentioned problems do not occur in the tapering of multimode fibers with cladding[5-6]. It has been reported that although the sensitivity decreases due to the remaining cladding, the device can be employed as an evanescent wave sensor using absorption spectroscopy of the external medium. However, to the best of our knowledge, the effects of the presence of fiber cladding, the refractive index of the external medium surrounding the tapering region on the transmission and sensing characteristics are unclear. The transmission properties of a multimode fiber taper has been studied based on the geometric optics [7-8]. It is known that geometric optics are no longer available for calculating the transmission properties if the coupling between modes occurs.

The geometric analysis method is not effective for examining when the dimensions of the tapered fiber core are reduced to the order of single mode fiber or when the structure of core and the input beam profile are complicated. In this paper, the beam propagation method, which is based on the wave optics approach instead of 
the geometric optics, was adopted to analyze highly biconically tapered cladded multimode fibers. Although the beam propagation method requires a lengthy calculation time for three dimensional structures, it is easy to apply to various device structures and beam profiles of the optical source. In this paper, the behavior of the biconically tapered cladded multimode fiber is discussed in terms of the guided mode theory. Transmission loss according to the tapering structure, refractive index of the surrounding medium, and wavelength were measured and analyzed. The feasibility of the biconically tapered cladded multimode fiber as a refractive index sensor was demonstrated.

\section{THEORETICAL CONSIDERATION}

Fig. 1 shows a schematic diagram of the biconically tapered cladded multimode fiber. Here $n_{c o}, n_{c}$, and $n_{e x}$ are the refractive indices of the core, cladding and surrounding medium, respectively. $a_{0}$ and $a_{w}$ are the core radii of a non tapered fiber and the waist of a tapered fiber, respectively. $b_{0}$ and $b_{w}$ are the cladding radii of a non tapered fiber and the waist of a tapered fiber, respectively. L denotes the taper length.

The transmission characteristics of the device were analyzed using a beam propagation method based on the wave optic approach. In the simulation, a step index multimode fiber with a core diameter, cladding diameter, refractive index of core, core-cladding refractive index difference, taper length(L) and wavelength of $50 \mu \mathrm{m}, 100$ $\mu \mathrm{m}, 1.46,0.02,2.5 \mathrm{~mm}$ and $1550 \mathrm{~nm}$, respectively, were considered. The following were assumed:

1. the device structure is symmetric in the longitudinal direction;

2. the core and cladding diameter of the multimode fiber were reduced at the same rate during tapering;

3. the fiber is fully illuminated and all modes are excited with uniform optical intensity; and

4. the phases of each mode are distributed randomly.

Fig. 2(a) and (b) show the procedure for beam propagation(left side) and the relative optical power(right side) along the fiber taper. It was assumed that the refractive index of the external medium was equal to that

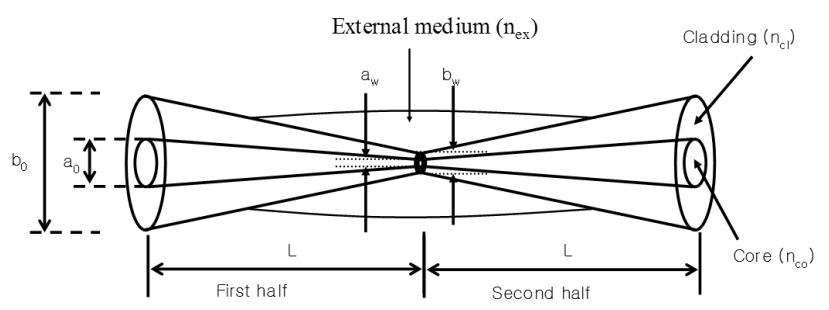

FIG. 1. Schematic diagram of the cladded multimode fiber biconical taper. of the fiber cladding. In this case, the device can be considered a biconically tapered multimode fiber without cladding because the refractive indices of the external medium and fiber cladding are equal. The total optical power confined in the core decreases gradually along the biconically tapered cladded multimode fiber. According to the guided mode theory, the total number of the guide modes is strongly dependent on the core diameter[9]. Higher order modes begin to leak into the cladding as the core diameter decreases along the fiber axis. Therefore, the transmission loss is somewhat inversely proportional to the tapering $\operatorname{ratio}\left(\frac{a_{w}}{a_{0}}\right)$.

When the refractive index of the external medium is less than that of the fiber cladding, the external medium plays the role of second cladding of the fiber taper. As a
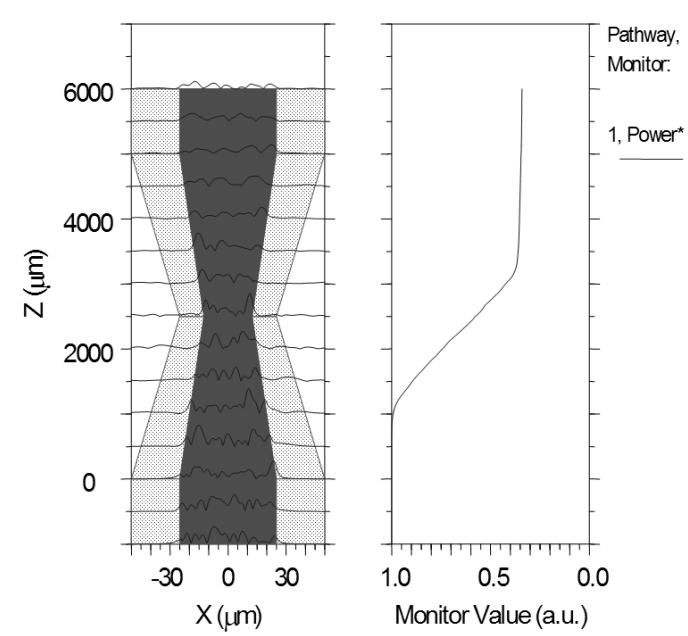

(a)
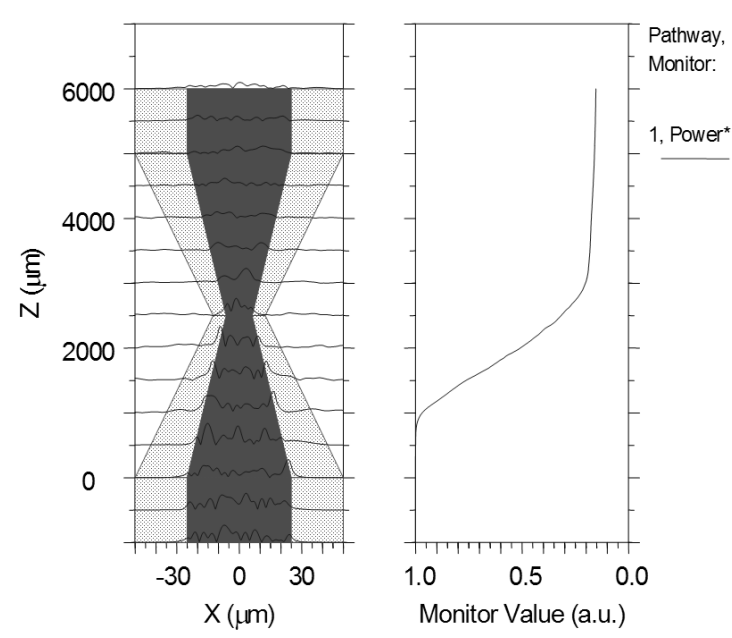

(b)

FIG. 2. Beam propagation(left) and optical power variation(right) along the fiber taper, (a) $\frac{a_{w}}{a_{0}}=0.5$, (b) $\frac{a_{w}}{a_{0}}=0.25$. 
result, there are two types of modes. One is the core mode, which is guided in the fiber core. The other is the cladding mode, which is confined to the fiber cladding. Some of the core modes are converted to the cladding mode with decreasing core size in the left side(first half region) of the tapered waist region. The cladding modes may re-couple into the core modes with increasing core size

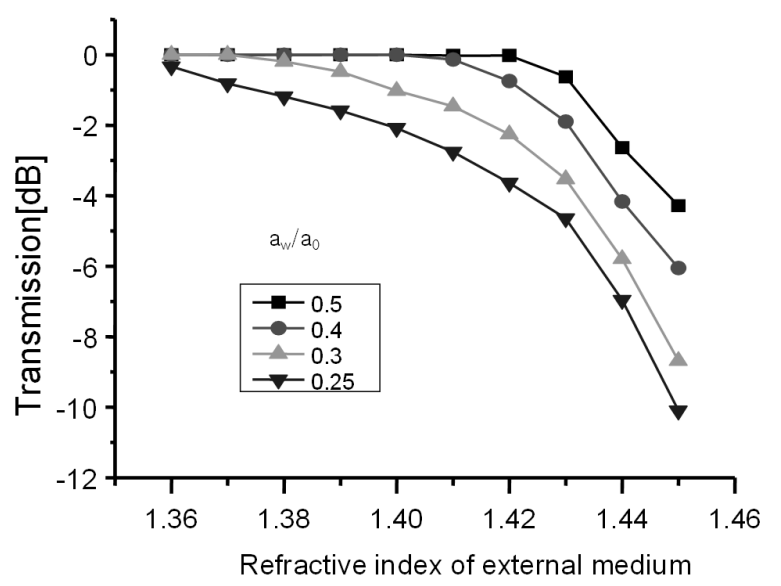

FIG. 3. Calculated transmission of the multimode fiber taper in accordance with the refractive index of the external medium.
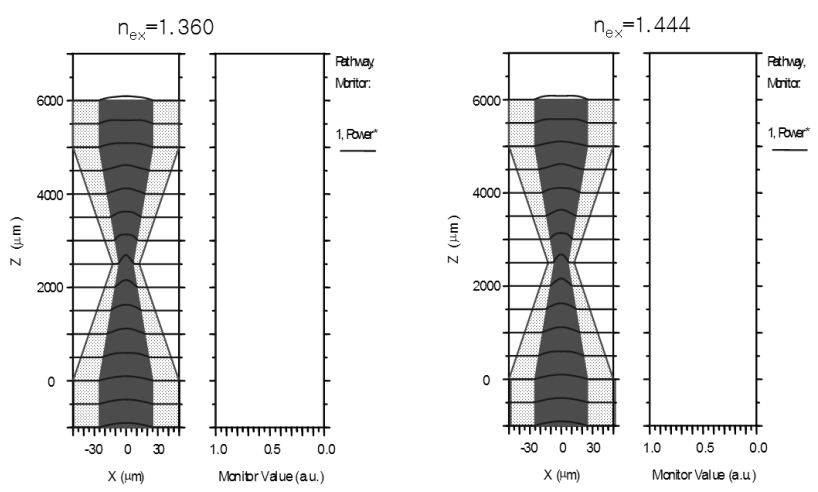

(a)
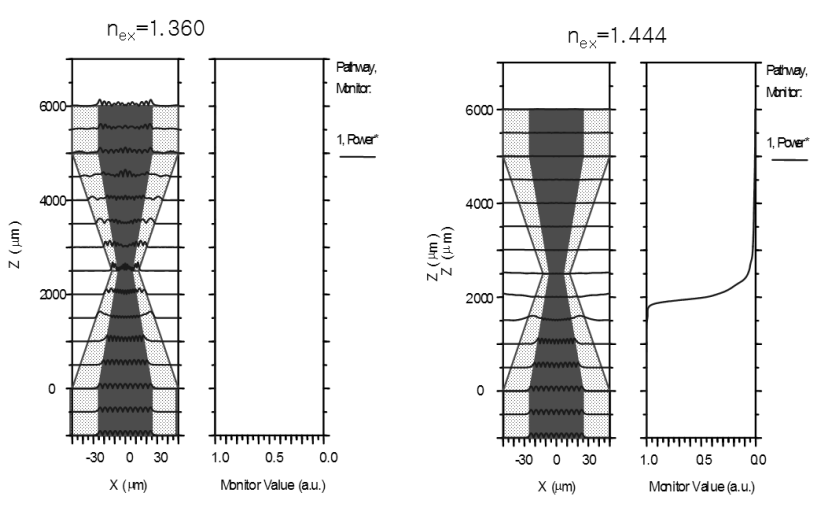

(c) in the second half region of the fiber taper according to the mode evolution process[10-12]. The re-coupling phenomenon does not occur in the biconically tapered multimode fiber without cladding.

Fig. 3 shows the dependence of the transmission on the refractive index of the external medium for several different tapering ratios. Here, the transmission indicates the optical power ratio between the input and output ends.

The simulation shows that the transmission is quite sensitive to the refractive index of the external medium. The dependence of the transmission on the $n_{\mathrm{ex}}$ of the biconically tapered cladded multimode fiber is more complicated than that of the uncladded device. In order to explain the behavior of the biconically tapered cladded multimode fiber, using the mode theory, the device was modeled into a two dimensional multilayer planar waveguide taper and the transmission characteristics for several modes were examined. Fig. 4 shows the beam propagation and optical power for several modes, including the fundamental mode, $5^{\text {th }}$ mode, $10^{\text {th }}$ and $15^{\text {th }}$ mode, were examined for two typical media with a high and a low refractive index, $\mathrm{n}_{\mathrm{ex}}=1.444$ and $\mathrm{n}_{\mathrm{ex}}=1.360$ with fixed tapering $\operatorname{ratio}\left(\frac{a_{w}}{a_{0}}\right)$ of 0.25

For the fundamental mode, an optical beam can pro-
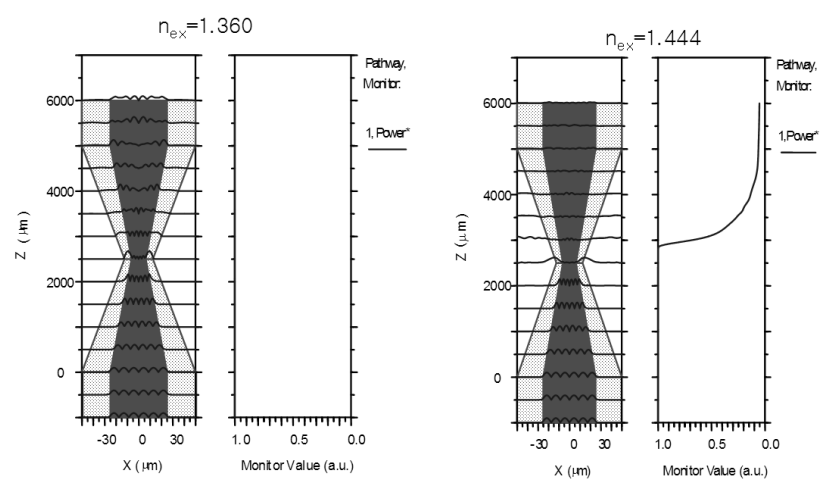

(b)
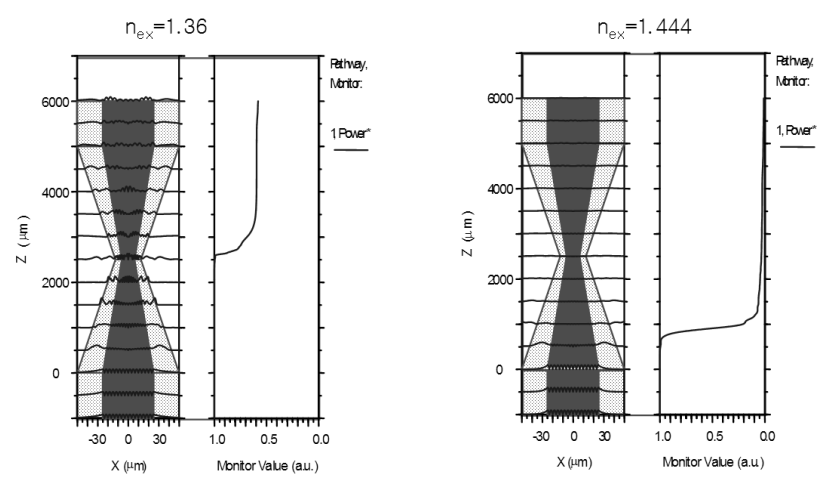

(d)

FIG. 4. Beam propagation and optical power variation for different modes; (a) fundamental mode, (b) $5^{\text {th }}$ order mode, (c) $10^{\text {th }}$ order mode, (d) $15^{\text {th }}$ order mode. 
pagate through the tapered region with negligible transmission loss regardless of the refractive index of the external medium, as shown Fig. 4(a). In this case, only the mode size changes. This suggests that the multimode fiber taper can be used for beam focusing of the Gaussian beam with the proper beam size. However, for the $5^{\text {th }}$ and $10^{\text {th }}$ mode, the transmission losses are different for the two cases, as shown Fig. 4(b) and 4(c). When $\mathrm{n}_{\mathrm{ex}}=1.444$, the fiber core no longer confines the modes as the core size decreases. As a result, the mode leaks into the external medium. The $10^{\text {th }}$ order has larger transmission loss than that of the $5^{\text {th }}$ mode. However, when $\mathrm{n}_{\mathrm{ex}}=1.36$, the lower refractive index of the external medium prevents the $5^{\text {th }}$ and $10^{\text {th }}$ modes from leaking, resulting in no transmission loss. In this case, the modes change from the core mode into the cladding modes at the right side of the taper. However, the cladding modes are still bound by the external medium. It is important that the optical power distributed in the fiber cladding be gradually confined into the core as the core size increases, as shown in Fig. 4(b) and (c). That is, the modes are converted from the cladding mode into the core mode again. It should be noted that the evanescent wave of the only cladding modes has access to the external medium. For the $15^{\text {th }}$ mode, the mode shows transmission loss, even though $n_{\mathrm{ex}}=1.36$, as shown in Fig. 4(d). The total number of the guided modes in the waist region of the fiber taper and the total transmitted power depends on the refractive index of the external medium when all the modes are excited. Therefore, it can operate as a refractive index sensor.

\section{EXPERIMENTS AND ANALYSIS}

A multimode fiber with a core diameter, cladding diameter and numerical aperture of $125 \mu \mathrm{m}, 50 \mu \mathrm{m}$ and 0.02 , respectively, was used for the experiments. The multimode fiber was heated with flames from two micro torches and was tapered by pulling. The core and cladding diameter was controlled by the tapering length. Two types of biconically tapered cladded multimode fiber with different tapering lengths(2L), $10 \mathrm{~mm}$ and $15 \mathrm{~mm}$, respectively, were prepared. Fig. 5 shows an image of the waist region of the multimode fiber taper. Practically, the shape of the fabricated tapered fiber is slightly different from the theoretical model. The tapering length $(2 \mathrm{~L})$ and tapering $\operatorname{ratio}\left(\frac{a_{w}}{a_{0}}\right)$ of the fabricated device are longer and larger than those of the theoretical model. In the center of the waist the cladding diameter was more smoothly changed than theoretical The cladding diameter was kept uniformly within several millimeters along the fiber direction in the center of the waist. After this process, the sample was mounted on a U shaped quartz tube, $40 \mathrm{~nm}$ in length, using an UV curing epoxy. Since one side of the quartz tube is opened, the
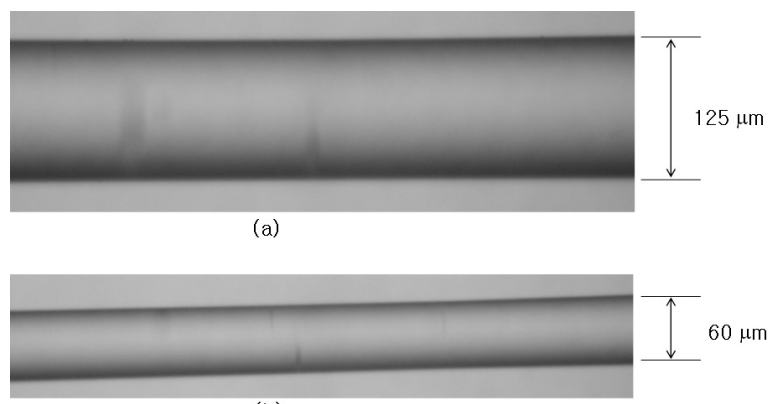

(b)

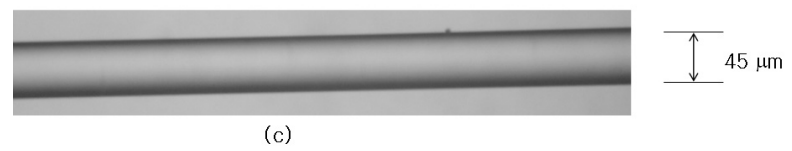

FIG. 5. Image of the waist of the multimode fiber taper, (a) before tapering, (b) $\mathrm{L}=5.0$ (c) $=7.5 \mathrm{~mm}$.

empty volume of the quartz tube can be filled with an external medium. Hence, the tapered region of the device is surrounded by the external medium. Several types of liquids were prepared as external media. The refractive indices of these materials were measured using a prism coupler at a wavelength of $1550 \mathrm{~nm}$. The prepared materials were made from water and glycerin. The experimental set-up consisted of a white light source made from a halogen ramp, DUT(device under test) and spectrum analyzer, which was used to detect the transmission dependence on both the external medium and wavelengths.

Fig. 6 shows the experimental results. In this experiment, the multimode fiber without tapering, whose length is equal to the DUT(device under unit), was used as a reference multimode fiber for the transmission. The transmission is the difference between the output spectrum of reference fiber and DUT. The higher tapered device shows higher transmission loss for the same external medium. As shown theoretically, the transmission loss increases with increasing refractive index of the external medium. Therefore, biconically tapered cladded multimode fiber may be used as a refractive index sensor. The experimental results also show the refractive index sensitivity increases with increasing wavelength. For a low refractive index external medium, such as air and water, the transmission loss is somewhat flat against the wavelength. On the other hand, for an external medium with a high refractive index, the transmission decreases with increasing wavelength. The wavelength dependence of the device in transmission is dependent on several parameters. The experimental results may be explained from the fact that the total number of modes guided in the fiber core and cladding at the waist region decreases step by step with increasing wavelength and refractive index of the external medium. Using the spectra corresponding to two refractive indices of external mediums 1.441 and 1.426 in Fig. 6(a), it can be estimated that near a wavelength 


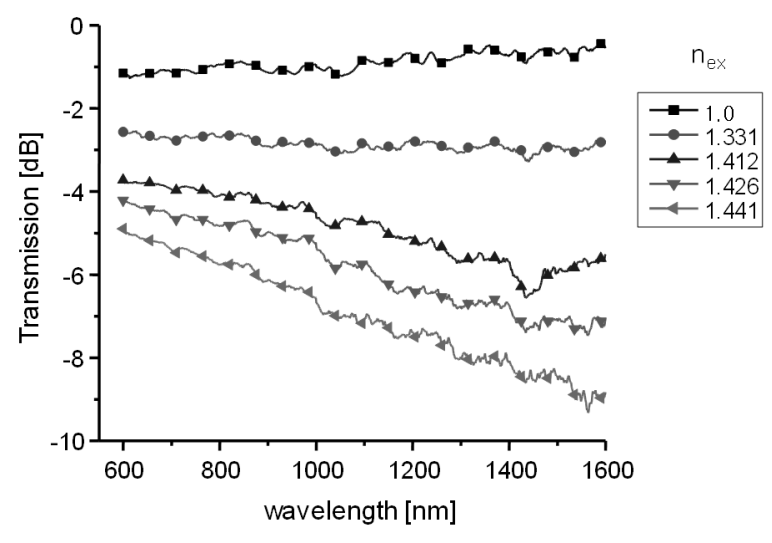

(a)

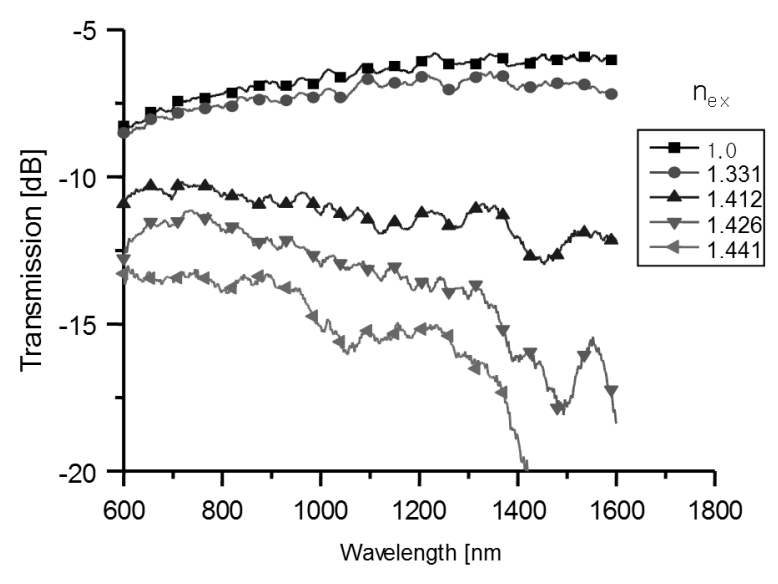

FIG. 6. The measured transmission of the biconically tapered multimode fiber, (a) $\mathrm{L}=5.0 \mathrm{~mm}$, (b) $\mathrm{L}=7.5 \mathrm{~mm}$.

of $1550 \mathrm{~nm}$, an about $3 \mathrm{~dB}$ change in transmission is made by a change of 0.015 in refractive index. Although the experimental results could not be properly compared with the simulation results due to the difficulties in measuring the exact taper, measured behaviors of the device agree with the theoretical expectations. In spite of the fact that the tapering ratio of the fabricated device $\left(\frac{a_{w}}{a_{0}}\right)$ was higher than the theoretical model, the measured transmission loss of the fabricated devices was higher as shown in Fig. 6(b). We thought that this result was attributed to the long tapering length of fabricated devices.

It was reported that unlike the biconically tapered cladded multimode fiber considered in this paper, the transmission of a single mode biconically tapered fiber device[13] using evanescent wave coupling with an external medium exhibits no leaky loss when the refractive index of the external medium is much lower than that of fiber cladding. it was demonstrated experimentally that the device can operate as a refractive index sensor for an external medium, especially for low index material.

\section{CONCLUSION}

The transmission and sensing characteristics of a biconically tapered cladded multimode fiber were analyzed using the beam propagation method. The effect of cladding on the transmission and sensing characteristics was explained in terms of mode coupling between the core and cladding modes. The transmission loss is dependent on the number of cladding modes bounded by the external medium. The transmission of the multimode fiber taper is quite sensitive to the refractive index of the external medium, which makes it possible to use as a refractive index sensor. The refractive index sensitivity is dependent on the device structure and optical wavelength. We observed that about $3 \mathrm{~dB}$ change in transmission was caused by a change of 0.015 in refractive index. In addition, we expect that the device can be also used for evanescent wave absorption sensor for spectroscopy because the cladding mode can be re-coupled into core mode.

\section{REFERENCES}

1. M. Tabib-Azar, B. Sutapun, and R. Petrick, "Highly sensitive hydrogen sensors using palladium coated fiber optics with exposed cores and evanescent field interactions," Sensors and Actuators B : Chemical 56, 158-163 (1999).

2. D. Luna-Moreno and D. Monzon-Hernandez, "Effect of the Pd-Au thin film thickness uniformity on the performance of an optical fiber hydrogen sensor," Applied Surface Science 253, 8615-8619 (2007).

3. S. T. Lee, R. D. Kumar, and P. S. Kumar, "Long period gratings in multimode optical fibers: application in chemical sensing," Opt. Comm. 224, 237-241 (2003).

4. R. Orghici, U. Willer, and M. Gierszewska, "Fiber optic evanescent field sensor for detection of explosives and CO2 dissolved in water," Appl. Phys. B : Lasers and optics 90, 355-360 (2008).

5. A. G. Mignani, "Evanescent wave absorption spectroscopy by means of bi-taped multimode optical fiber," Applied Spectroscopy 52, 546-551 (1998).

6. S. Guo and S. Albin, "Transmission property and evanescent wave absorption of cladded multimode fiber tapers," Opt. Exp. 11, 215-223 (2003).

7. Y.-F. Li and J. W. Y. Lit, "Transmission properties of a multimode optical-fiber taper," J. Opt. Soc. Am. A 2, 464-468 (1985).

8. Y. Yang, J. Lee, K. Reichard, P. Ruffin, F. Liang, D. Ditto, and S. Yin, "Fabrication and implementation of a multi-to-single mode converter based on a tapered multimode fiber,” Opt. Comm. 249, 129-137 (2005).

9. G. Keiser, Optical Fiber Communications (McGraw-Hill, USA, 1983), Chapter 2.

10. A. J. Fielding, K. Edinger, and C. C. Davis, "Experimental observation of mode evolution in single-mode tapered optical fibers," J. Lightwave Tech. 17, 1649-1656 
(1999).

11. A. C. Boucouvalas and G. Georgiou, "Tapering of single -mode optical fibers," IEE proceedings J, Optoelectronics 133, 385-392 (1986).

12. A. Yacoubian, W. Lin, and J. H. Bechtel, "Fabrication of polymer waveguide tapers to minimize insertion loss," Proc. SPIE 4490, 22-31 (2001).

13. K. T. Kim and K. H. Park, "Fiber-optic temperature sensor based on single mode fused fiber coupler," J. Opt. Soc. Korea 12, 152-156 (2008). 